\title{
Rethinking the formula
}
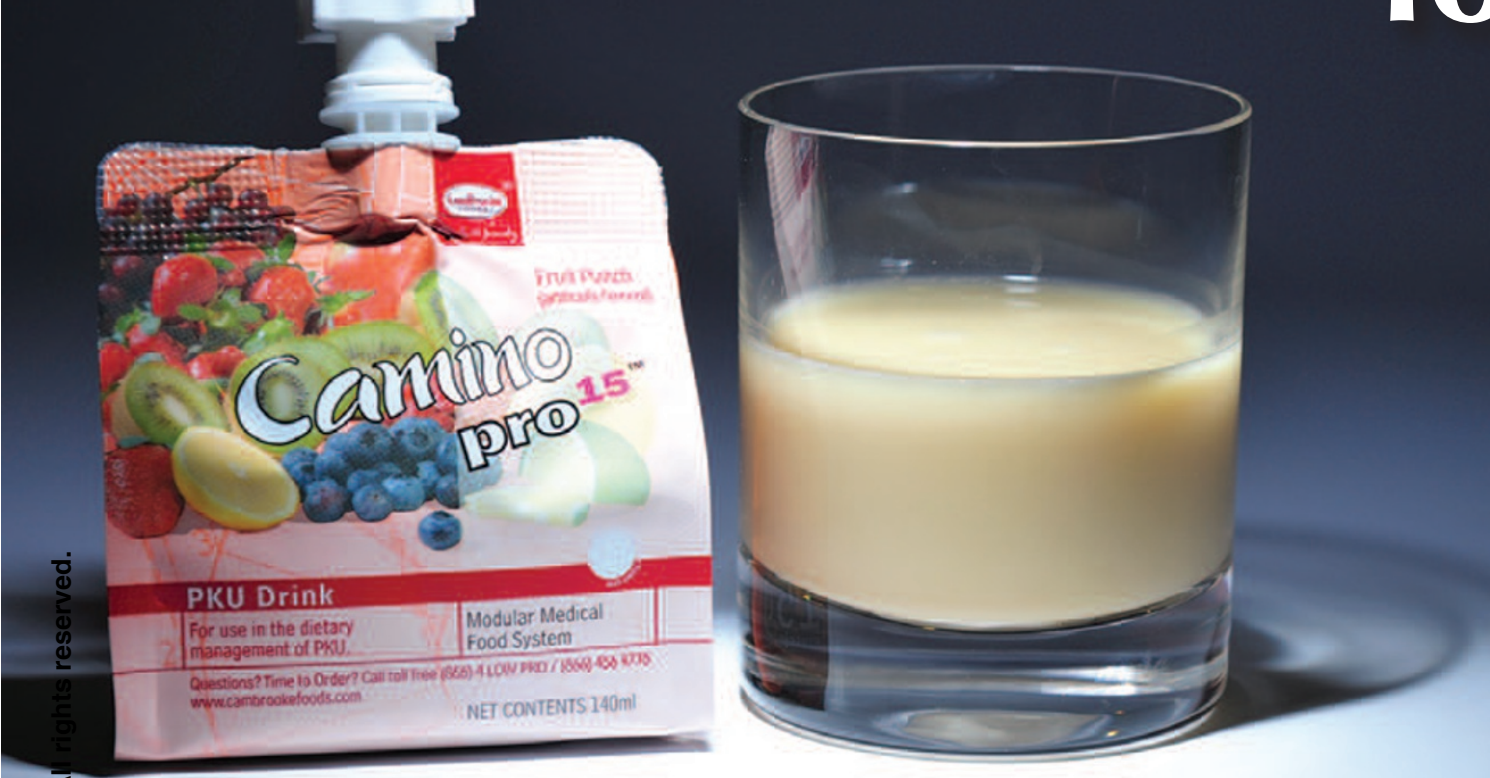

Health insurance covers drugs approved by regulatory agencies, but it often doesn't pay for the products known as 'medical foods' needed to keep individuals alive and well. This lack of reimbursement means that many who cannot afford these life-saving diets suffer brain deterioration and disability-or worse. Roxanne Khamsi reports on the battle for medical foods and how it could affect the treatment of diseases as diverse as osteoporosis and Alzheimer's.

Katie Maguire does her best to explain the kind of sandwich she wants. Looking up from her seat at Balthazar, a bustling restaurant amid the cobblestone streets of Manhattan's trendy SoHo district, she smiles at the waiter and asks for a chicken club-one without any chicken or bacon. "Can I just have a sandwich, just lettuce and tomato?" she asks. Some confusion ensues, and ultimately she tries a different description: "A BLT with no bacon." The waiter finally nods his head and scribbles down the order.

Although Maguire eats like a vegetarian, she does not do so for philosophical reasons. Her reason for skipping meat is a genetic condition whereby her body cannot tolerate more than a few grams of proteins each day. At age 28, Maguire has never eaten a hamburger.

Because Maguire can't process protein normally, she travels with a stash of special products to keep her well fueled. They contain carefully formulated mixtures of the protein building blocks known as amino acids that her body can tolerate. Without these special formulas, adults who have the disorder that affects Maguire, known as phenylketonuria, or PKU, develop cognitive problems ranging from short-term memory loss to hallucinations. Women with her disorder who forgo these tailored sources of amino acids give birth to babies with permanent developmental handicaps, including severe mental retardation.

Yet, even though Maguire relies on amino acid formulas to avoid cognitive deterioration, the US Food and Drug Administration (FDA) does not classify such products as drugs. Instead, they are considered 'medical foods'. That key distinction has made it much harder for Maguire, and others like her, to obtain insurance coverage for these expensive products, which can cost upward of $\$ 10,000$ a year.

Michael Watson, executive director of the American College of Medical Genetics and Genomics (ACMG) in Bethesda, Maryland, describes the realm of medical foods as a "regulatory no-man's land," a confusing gray zone of peculiar rules. For example, the FDA does not ask for a new drug application, or NDA, for medical foods, nor does it require the extensive clinical trials necessary for drug approvals. But the agency does stipulate that medical foods should be made according to guidelines known as good manufacturing practice and obtained with the consent of a doctor. (A third product category, known as 'dietary supplements', requires no such physician supervision.)

Whether an item gets classified as a drug or a medical food is not always predictableor permanent. "It's a mess," Watson says. "The FDA doesn't know where to draw the line."

The ambiguity around the regulation and reimbursement of medical foods is a growing problem-and not just for the tens of thousands of people in the US living with so-called 'inborn errors of metabolism, including the estimated 14,500 individuals with PKU. There are now medical foods available for conditions ranging from heart disease to osteoporosis to HIV/AIDS. And the list of products is increasing: in 2009, the FDA gave the green light to a new medical food known as Axona for people with Alzheimer's disease. 
Maguire, a first-grade teacher, ultimately prevailed in obtaining reimbursement for the formula she needs thanks to the help of the staff of then-New York attorney general Andrew Cuomo (now governor of $\mathrm{New}$ York). But others aren't as lucky. They give up on fighting their insurance companies or lack coverage to begin with, and they simply cannot pay out of pocket. "A lot of people can't afford these special diets," says Alex Kemper, a pediatrician at the Duke University Medical Center in Durham, North Carolina.

Those who are earning too much to receive government assistance yet are too poor to pay out of pocket suffer the most, according to Cynthia Le Mons, executive director of the National Urea Cycle Disorders Foundation, who lost both a sister and a son to a metabolic disorder known as ornithine transcarbamylase deficiency, which can prove particularly lethal in infants. "If you're a family where you fall into that donut hole, your child does not have a good outcome," she says. "And that just should not happen in the United States of America."

\section{Happy anniversary}

The fact that Katie Maguire is a healthy adult today is in large part thanks to Robert Guthrie, a physician-scientist with a love of sailing and a penchant for sleeping in the lab, who developed the newborn screening test for PKU in 1957. This year marks the fiftieth anniversary of the passage of state laws in Oregon and Massachusetts to mandate this test for all babies-the first such requirements of now-widespread newborn screening in the US.

The assay works by gauging the levels of the amino acid phenylalanine in the blood. At some point soon after she was born, a doctor or nurse pricked Maguire's heel to take a blood sample. A lab analysis based on Guthrie's method then showed that her phenylalanine levels fell above the normal range of 0.5 to 2.0 milligrams per deciliter of blood, indicating a malfunction in her body's version of the liver enzyme known as phenylalanine hydroxylase (PAH).

About 1 in 50 people in the US carry a mutation in the gene for this enzyme, but a second, functioning version keeps them healthy. By contrast, individuals with PKU have two faulty copies of the $P A H$ gene, meaning that they have no working enzyme to convert phenylalanine into tyrosine, another amino acid. Here's the rub: tyrosine is a precursor for neurotransmitters such as dopamine-crucial for normal brain maturation and mood regulation. If untreated, a baby born with PKU will suffer severe

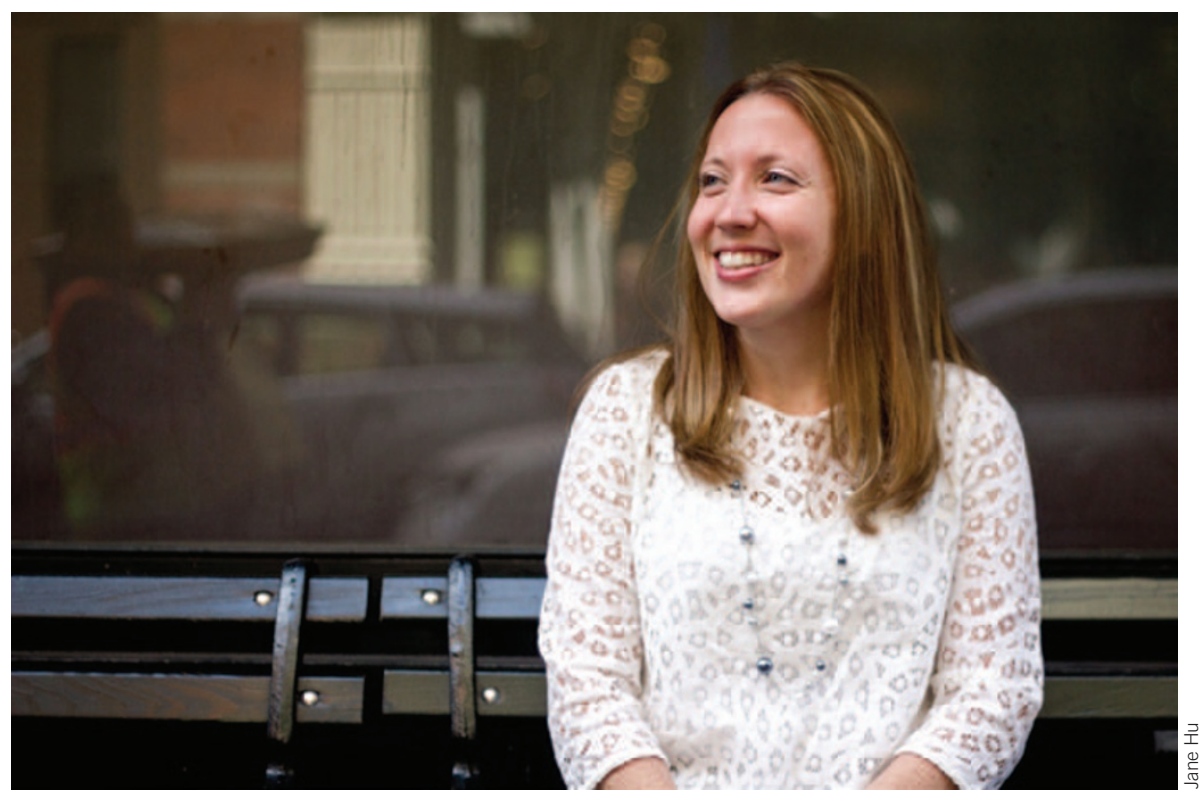

Teaching hope: Katie Maguire has fought for reimbursement and won.

mental retardation and other developmental problems.

Virtually every known protein in food contains a phenylalanine component. So doctors must immediately start babies born with PKU on a phenylalanine-free formula made up of other amino acids, including tyrosine. Evidence is emerging that individuals need to stick to this regimen for their entire lifetimes. One study has found that people with PKU who discontinue the special diet by age 10 have a 12-point drop in their IQ by adulthood, on average ${ }^{1}$. These individuals are also at risk of developing delusions and other symptoms similar to schizophrenia or bipolar disorder, often necessitating antipsychotic drug treatments or tranquilizers.

Staying on the diet is no easy task, though. For one thing, the smell of the formula is pungent, and it has the tang and consistency of sour milk. The liquid, and the foods modified to be low in protein for people with $\mathrm{PKU}$, leave a long-lasting bitter taste at the back of the tongue that water does not quickly wash away. It's no surprise why people like Maguire drift off of the diet in their early teens, when the frustration of carrying and consuming the unpalatable and odorous formulas interferes with the pressures and norms of high school (never mind matters related to cost).

In the teenage years during which Maguire gave up on consuming formula, her family watched as her mood-and her gradesdeteriorated. She was prone to emotional outbursts and struggled to achieve Bs on her report cards. Just before heading to college, her doctor gave her a tough talk, urging her to get back on the diet. The conversation shook Maguire out of her complacency, and as a college freshman she transitioned back onto the formula regimen.

As the physician had predicted, her grades started to improve. "I started feeling better. I was able to think back and be like, 'Oh my God, I was like that?" recalls Maguire, her eyes widening. "You know, once you're out of that fog, you realize what it was like to be off diet. When you're going through it, you don't have any idea."

\section{Paying the price}

The annual wholesale cost of basic formula for an adult on a 2,000-calorie-a-day diet is more than $\$ 8,500$ (ref. 2). However, this amount excludes the cost of their other dietary needs, and price markups of as much as $300 \%$ at the point of distribution are common. What's more, foods modified to be low in proteinso that they are safer for people with PKU to eat-are generally priced two to eight times higher than their regular supermarket counterparts, and they usually have associated shipping costs. Whereas a run-of-the-mill loaf of bread costs less than $\$ 3$, the same amount of bread specially manufactured for people with PKU can cost more than $\$ 13$.

Nutritionists and doctors have numerous stories of paying out of pocket to help families that cannot afford formula or modified foods. "I've walked to the drugstore to buy supplemental formula for patients in the past," says Jerry Vockley, chief of medical 
genetics at the Children's Hospital of Pittsburgh.

To complicate matters in the US, the reimbursement requirements for PKU formula vary from state to state. At present, there are 38 states with laws or programs that cover PKU, but some ensure access only until age 18 or 21 . A few years ago, Maguire, who lives in the suburban community of Cornwall, New York, had considered following in the footsteps of a cousin who relocated to North Carolina. But she nixed her plans to move when she learned that the state doesn't mandate medical food reimbursements for adults with PKU. "In the end, I didn't go because there was no coverage there," Maguire says. "Sometimes I feel like I'm trapped here."

Even in states like New York that mandate coverage, insurance programs known as 'self-insured plans' are exempt from having to provide it. A self-insured health plan is defined as a customizable arrangement in which an employer chooses to set up a fund and assumes the risk of paying for the health claim costs of its employees. For large companies, it's typically a cheaper option than regular insurance. And, in these tough economic times, such plans are on the rise: a November 2012 report by the nonprofit Employee Benefit Research Institute found that the proportion of US workers in privatesector self-insured health plans increased from $41 \%$ in 1998 to $59 \%$ in 2011 . worked as a teacher had exactly this type of self-insured plan-and to her chagrin it had decided it would opt out of the New York State requirement that insurance companies reimburse all individuals with PKU for formula expenses. She pleaded her case to plan administrators for many months with letters and calls. It was only after she convinced Cuomo's office to take her side that the company finally gave in to the pressure.

Like self-insured plans, the Federal Employees Health Benefits program is
The school system for which Maguire has

exempt from certain state requirements. That is why Jennifer Payne, a 39-year-old who has PKU and works as a pharmacist at a medical center run by the US Department of Veterans Affairs in Baltimore, does not receive coverage for her formula through her employer despite a 1995 Maryland law that ensures insurance coverage of low-protein foods and medical foods for all people with inherited metabolic diseases. "The federal government fails to comply with the good and acceptable practice guidelines for the treatment of PKU as modeled by the state of Maryland, in which I live," Payne says.

Given the unwieldy patchwork of coverage, patient advocates have tried to get the US Congress to pass the Medical Foods Equity Act, first proposed in 2009. "We'd like to see a federal law that would require insurance companies and the government to ensure lifetime coverage," says Christine Brown, a mother of two children with PKU and the executive director of the National PKU Alliance, located in central Wisconsin. The bill would mandate that private insurers and federal health programs cover formula for inborn errors of metabolism, such as PKU, and it would also bar exemptions for this by self-insured plans.

Le Mons sees the legislation as essential and notes that, for conditions such as ornithine transcarbamylase deficiency, access to formula can make the difference between life and death. But with one of the main sponsors, former Massachusetts senator John Kerry, having been promoted out of Congress to the President's cabinet, and the Capitol in perpetual legislative gridlock, she worries that the bill won't move ahead anytime soon.

In the meantime, families continue to call for broadened coverage of formulas for other inborn metabolic disorders at the state level. Nancy Mahmoud, the mother of a child with eosinophilic gastrointestinal disorder, urged Connecticut lawmakers to do just this in a recent letter to the editor in the Westport

Costs of selected foods modified to be low in protein and their regular counterparts

\begin{tabular}{|c|c|c|c|}
\hline Regular food option ${ }^{a}$ & $\begin{array}{l}\text { Cost per } 100 \mathrm{~g} \\
\text { product in dollars }\end{array}$ & Low-protein version & $\begin{array}{l}\text { Cost per } 100 \mathrm{~g} \\
\text { product in dollars }{ }^{\mathrm{b}, \mathrm{c}}\end{array}$ \\
\hline Spaghetti & 0.37 & Aproten low protein pasta & 2.20 \\
\hline Flour & 0.17 & Wel-plan baking mix & 1.29 \\
\hline Bisquick $^{\circledR}$ & 0.31 & $\begin{array}{l}\text { Taste connections low protein } \\
\text { baking mix }\end{array}$ & 0.58 \\
\hline Crackers & 0.64 & Loprofin crackers & 1.94 \\
\hline Tortillas & 0.40 & Low-pro tortillas & 2.04 \\
\hline Peanut butter & 0.70 & Low-pro peanut spread & 1.94 \\
\hline
\end{tabular}

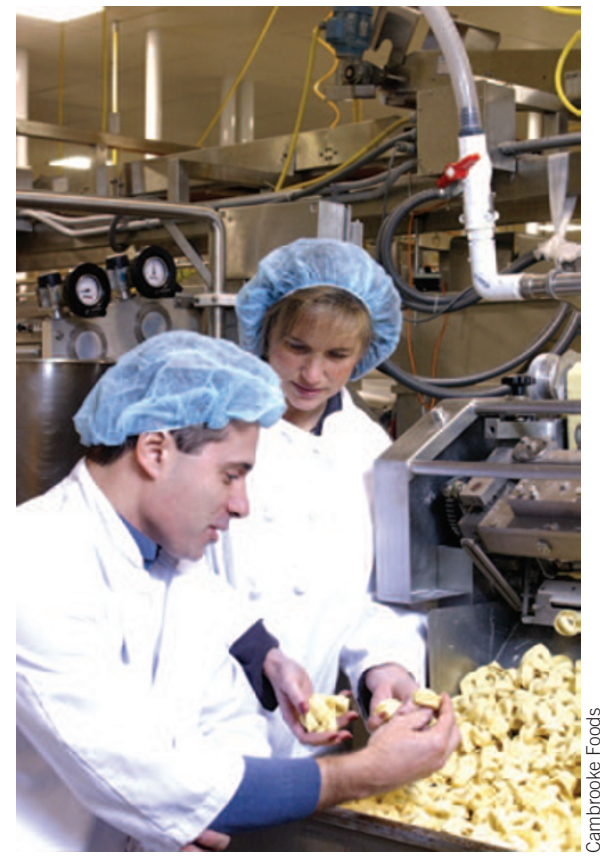

Blast from the pasta: The Paolellas in 2003.

News, citing dire circumstances: "There is now an underground market where parents who have coverage sell their extra cans of medical food under the table to those who don't have coverage," she wrote of the situation.

\section{A drug by any other name?}

Even if state or federal governments fail to pass new legislation on formula coverage in the near term, people living with PKU still hope that the FDA might reclassify these products as drugs, thereby compelling insurers to pay for them. It's not as unreasonable a hope as it sounds: until the 1970s, the FDA had actually classified the formula available for PKU at the time, Lofenalac, as a drug.

The manufacturer of Lofenalac, Mead Johnson, then based in Indiana, had been producing the formula since 1958 . When the FDA changed its position in 1972 and subsequently said that products like Lofenalac would, going forward, be seen not as drugs but rather as foods "for special dietary use," this loosened the regulatory requirements for bringing formulas for metabolic disorders to the market.

The idea was to encourage companies to innovate and provide for a small patient population. "A decision was made that if you want to have more of these formulas, you have to have some flexibility to do that," Vockley says. Today, there are at least five companies that have PKU formulas on the market in the US, including Ohio's Abbott Nutrition and Cambrooke Foods, headquartered in central Massachusetts. 
State mandates for medical food coverage in the US

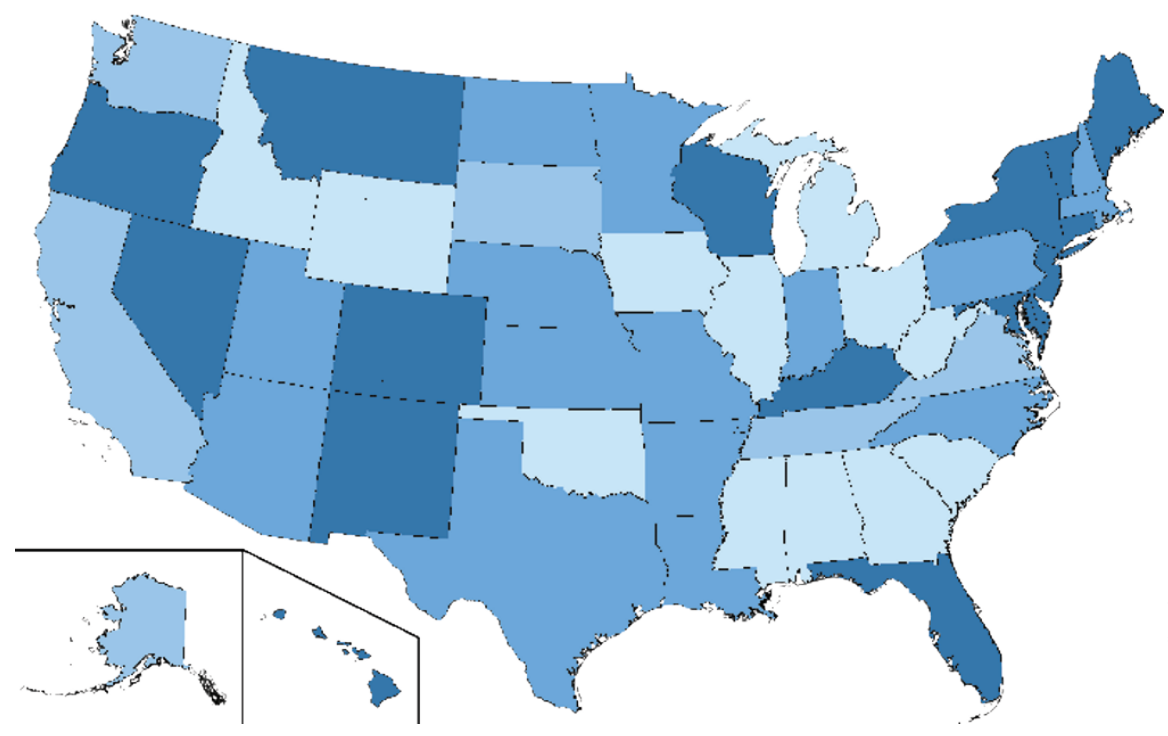

No mandate*

Select inherited metabolic disorders

Only for PKU (age limits vary)

All inherited metabolic disorders

*Wyoming will provide medical food coverage for all inherited metabolic disorders when an act goes into effect on 1 July 2013.

People with PKU appreciate that they now have a wider selection of products thanks to the 1972 reclassification of PKU formula. But the reimbursement consequences still rankle them. "As a result of this decision, the FDA has left a 40-year financial burden on PKU patients and their families because insurance companies continue to deny access to this costly treatment and refuse to reimburse patients for this product, which is still defined as a food," Payne, the Baltimore pharmacist, wrote in an open letter to the agency last summer. "I consider Lofenalac a drug because it saved me from a life of mental retardation and institutional care."

The formal definition of a 'medical food' did not come until 1988, when Congress introduced it as part of amendments enacted to the Orphan Drug Act. The definition stipulates that such a product, among other things, "is intended for the specific dietary management of a disease." Further clarifications came over the following decade, including rules published by the FDA in 1993 that outlined, for example, that a medical food should be given under medical supervision.

As a rule of thumb, companies seeking to get a new therapeutic agent approved as a drug must always submit an NDA to the FDA based on clinical trials. But since PKU formulas can be seen by the agency as ways of "managing" a disease, rather than treating the ailment, the agency does not ask for an NDA in these cases. The company can then decide to put its product on the medical-food path.

The notion that a formula could get approved as a drug is not impossible. "If a product is appropriately marketed as a medical food but the firm wants to pursue drug marketing for the product, the firm would have to meet all of the regulatory and legal requirements to get that product on the market as a drug," Benson Silverman, head of the FDA's Infant Formula and Medical Foods staff, told Nature Medicine in an email from the agency.

Medical-food makers don't eschew the concept of clinical trials per se. In fact, a key component of many of Cambrooke's products has undergone investigation in a small-scale human study. The ingredient is a protein found in milk whey known as glycomacropeptide (GMP) - the only known naturally occurring dietary protein that lacks phenylalanine. This unique feature of GMP was struck upon in the 1990s by a food scientist in Wisconsin, and the protein subsequently underwent preclinical testing. A study conducted a few years ago with funding from the US National Institutes of Health tested a formula containing GMP on 11 people with $\mathrm{PKU}$ for four days ${ }^{3}$. It found hints of better protein retention with the GMP diet compared with the standard amino acid formula, perhaps because the body might processes it more slowly than individual amino acids.

Researchers are now enrolling 30 people for a follow-up trial in which the participants will consume products that contain GMP for three weeks. The study will measure the volunteers' cognitive performance as well as their blood phenylalanine levels. The FDA has informed the scientists that they can pursue the medical-food path, and it will not require the paperwork that would be necessary for a drug trial.

Even supposing that small companies such as Cambrooke had the resources and desire to pursue drug status for their products, they would face the prickly problem of how to conduct the massive placebo-controlled randomized trials that are the gold standard for new medicines, explains Cambrooke chief executive David Paolella, an architect who cofounded the business with his wife Lynn, a jeweler, after two of their children were born with PKU. First, since foods containing phenylalanine cause detrimental cognitive effects, it would be unethical to place a person with PKU on a placebo course in which they derived their protein calories from meat or regular bread. And second, the unique taste and odor of the special solid foods and formulas that lack this amino acid are a dead giveaway, making it difficult to create blinded treatment protocols. "This is the dilemma the entire industry sits in," says Paolella.

\section{Food for thought}

The questions about what defines a medical food will likely grow as the market does-and that market now extends far beyond PKU and other inherited metabolic disorders. Vitamax, for example, is a chewable tablet designed to meet the specific vitamin needs of people with cystic fibrosis; Vascazen, meanwhile, contains purified omega-3 fatty acids for people with cardiovascular disease; Abbott Nutrition's Juven provides nutrients to people with HIV or AIDS experiencing excessive weight loss due to disease; Limbrel capsules, from Arizona-based Primus Pharmaceuticals, serve as a medical food for osteoarthritis. The list goes on.

All told, analysts estimate that more than 300 medical foods have launched since 2009 , with the global market for such products surpassing $\$ 9.3$ billion per year. US sales of medical foods hit $\$ 2.1$ billion in 2011 and are growing at approximately $10 \%$ annually, according to bioStrategies Group, a Chicagobased consulting group.

In the US at present, about 45,000 people with Alzheimer's receive the medical food 
A comparison of medical foods versus other types of foods or drugs in the US

\begin{tabular}{|c|c|c|c|c|}
\hline \multirow[t]{3}{*}{ Characteristic } & \multicolumn{4}{|c|}{ Intervention type } \\
\hline & \multicolumn{3}{|c|}{ Food } & \multirow[t]{2}{*}{ Drug } \\
\hline & Conventional food & Dietary Supplement & Medical Food & \\
\hline $\begin{array}{l}\text { Can be source of nutritional } \\
\text { needs }\end{array}$ & Yes & No & Yes & No \\
\hline Must be formulated & No & No & Yes & No \\
\hline Requires medical supervision & No & No & Yes & Yes \\
\hline $\begin{array}{l}\text { US FDA approval needed } \\
\text { before marketing }\end{array}$ & No & No & No & Yes \\
\hline Health claim labeling & Nutrition facts & Supplement facts & Nutrition facts & Package insert \\
\hline
\end{tabular}

Axona under the supervision of a doctor, according to its maker, the Colorado startup Accera. The product-a vanilla-flavored powder that is mixed into drinkable shakeworks when it is converted by the liver into ketone bodies, an alternative fuel to glucose. This is the key, because the brain cells of people with Alzheimer's cannot efficiently metabolize glucose.

Data from a 152-person trial showed that a 90-day course of Axona resulted in a 3-point improvement on a 70-point scale measuring cognitive function among individuals with mild-to-moderate Alzheimer's who lack the APOE4 genotype; those on placebo experienced a 2-point decline over the same period (Henderson, S.T., et al., Nutr. Metab. $\mathbf{6 , 3 1}, 2009$ ). Since its launch in 2009, doctors have begun introducing Axona to their patients. "I have about 200 or so people on it," says James Galvin, a geriatric neurologist at the New York University Langone Medical Center, who serves as a paid consultant to Accera. "A little over $50 \%$ get it covered by medical insurance," he notes. The product can cost as much as $\$ 100$ a month at a retail pharmacy.

Last summer, Nestlé Health Science, a subsidiary of the Swiss giant Nestlé, acquired a stake in Accera to advance its products in clinical trials. Accera is now actively recruiting participants for a 480-person trial of a new, easier-to-digest formulation of caprylic triglyceride, the most active ingredient in Axona, under an investigational new drug protocol. The company hopes that the study will provide evidence of a strong-enough benefit to get the novel product approved by the FDA as a drug.

Likewise, a three-year-old biotech startup called Ultragenyx Pharmaceutical Inc. also has products in its pipeline that sit at the boundary of what defines a medical food versus a drug. The California company's lead therapeutic, UX001, is an extendedrelease tablet of sialic acid undergoing phase 2 drug testing for a rare muscle-wasting disorder known as hereditary inclusion body myopathy. Sialic acid is a naturally occurring simple sugar, currently available as a health supplement.

\section{A dose of reality}

It's unlikely to see companies trying to reformulate medical foods for PKU to test them as drugs anytime soon. Notably, though, the PKU community has started to see some more traditional pharmacological developments. In 2007, the FDA approved sapropterin dihydrochloride, a pill marketed as Kuvan by California's BioMarin Pharmaceutical Inc. For about $2-3 \%$ of people with PKU who have specific mutations in phenylalanine hydroxylase, the so-called 'chaperone molecule' drug coaxes the enzyme to achieve proper function, allowing these individuals to eat whatever they want. In many other people with the disorder, the drug enables them to introduce a few more grams of protein into their daily diets-a small but significant improvement.

Within the next few years, experts also anticipate an enzyme-replacement drug known as Peg-Pal-short for PEGylated recombinant phenylalanine ammonia lyaseto receive market approval for patients with PKU. The medicine, slated to enter phase 3 trials within the next couple of months, could theoretically allow all individuals with PKU to eat regular foods.

Regardless of the promise of injection therapies such as Peg-Pal, people with PKU say that access to formula will remain crucial. Maguire points to a friend currently enrolled in the Peg-Pal trial who ran into a tough situation last fall. "She lives in Long Island and typically comes to Manhattan for a Peg-Pal injection," Maguire says. "Hurricane Sandy hit, and she couldn't get to New York City to receive Peg-Pal." It took a few weeks to get access to the drug. In the meantime, "she couldn't get her formula, she had no low-protein foods, and she has three young children," Maguire recounts. Her friend's phenylalanine levels shot up, and as a result "she was having panic attacks."
The controversy regarding medical food coverage will probably remain as issue for years to come. Rani Singh, a genetic nutritionist at the Emory University School of Medicine in Atlanta and a leading advocate for patients with PKU, believes that insurance companies are loath to broaden coverage for medical foods generally. "What they're worried about is obesity and people getting insurance payments for their Slim-Fast," she says.

The American College of Medical Genetics and Genomics is in the midst of developing new PKU practice guidelines, due out in the next few months. The guidelines as currently drafted recommend lifetime therapy for PKU, which would pressure medical insurance providers to cover formula and special foods, regardless of a person's age.

What continues to astonish the medical community is that insurance providers seem more willing to reimburse for medicines such as Kuvan, which can cost upward of $\$ 80,000$ a year, because of their designations as drugs than to do so for a dietary formula. "Ironically, we get better coverage for Kuvan from private insurance companies than we do for medical foods," which cost far less, says Debra Hook, a metabolic dietitian at the Children's Hospital Los Angeles.

Until PKU formulas receive better coverage by insurance companies, people with the disorder who cannot pay out of pocket will see their health deteriorate, some to the point of having to undergo hospitalization due to bipolar or schizophrenic episodes, Hook notes. The cost calculus by insurers simply does not make sense, she says: "One hospital visit costs so much more than the medical foods do."

Roxanne Khamsi is chief news editor at Nature Medicine in New York and is online at@rkhamsi.

1. Koch, R. et al. Eur. J. Pediatr. 155, S90-S92 (1996).

2. Camp, K.M., Lloyd-Puryear, M.A. \& Huntington, K.L. Mol. Genet. Metab. 107, 3-9 (2012).

3. van Calcar, S.C. et al. Am. J. Clin. Nutr. 89, 1068 1077 (2009). 